\title{
Time course of grouping of shape by perceptual closure: Effects of spatial proximity and collinearity
}

\author{
Bat-Sheva Hadad and Ruth Kimchi \\ University of Haifa, Haifa, Israel
}

\begin{abstract}
We examined time course of grouping of shape by perceptual closure in three experiments using a primed matching task. The gaps between the closure-inducing contours varied in size. In addition, depending on the distribution of the gaps along the closure-inducing contours - occurring at straight contour segments or at the point of change in contour direction - collinearity was either present or absent. In the absence of collinearity, early priming of the shape was observed for spatially close fragments, but not for spatially distant fragments. When collinearity was available, the shape of both spatially close and spatially distant fragments was primed at brief exposures. These results suggest that spatial proximity is critical for the rapid grouping of shape by perceptual closure in the absence of collinearity, but collinearity facilitates the rapid grouping of shape when the closureinducing fragments are spatially distant. In addition, shape priming persisted over time only when the collinear fragments were spatially close, suggesting that a stable representation of shape depends both on collinearity and spatial proximity between the closure-inducing fragments.
\end{abstract}

Upon viewing visual scenes, the observer is often confronted with fragmented visual information. Contours are routinely fragmented by occlusion, shadows, and lowreflectance contrast, yet the perceived visual world contains coherent objects and continuous surfaces. The Gestalt psychologists have long recognized the problem of perceptual organization and proposed a set of principles guiding the perceptual integration of visual elements by virtue of certain properties that are present in the image, including proximity, similarity, good continuation, common fate, and closure (Wertheimer, 1923/1955). Furthermore, they explicitly noted the crucial role of closure in perceptual organization, and, in particular, in the formation of shape (Koffka, 1935).

Later, several psychophysical studies documented the role of closure in perceptual organization (e.g., Elder \& Zucker, 1993, 1994, 1998; Kimchi, 2000; Kovács \& Julesz, 1993; Marino \& Scholl, 2005; Saarinen \& Levi, 1999). For example, Kimchi (2000) found that grouping by closure occurs early and rapidly, Kovács and Julesz demonstrated that contour detection sensitivity is greater for fragmented closed contours (against a cluttered background) than for open contours, Saarinen and Levi showed that shape discrimination is more precise for closed contours than for nonclosed contours, and Elder and Zucker (1993) showed that search for a concave target among convex distractors is efficient for closed stimuli but inefficient for open ones.

In natural scenes, however, closed contours often have gaps that vary in size, and, depending on the distribution of gaps along the contour, collinearity may be present or absent in the image. Indeed, several studies suggest that the utilization of perceptual closure for grouping of shape varies with the degree to which the closure-inducing contours are spatially close. This was first demonstrated by Gillam (1975), who found that the degree to which two ambiguously rotating lines were perceived as a unit decreased monotonically as a function of the gap size between closure-inducing elements added to these rotating lines. Extending this finding, Elder and Zucker (1993) showed that target detection time for fragmented stimuli decreases monotonically as the length of the connecting segments increases. On the basis of these findings, it has been suggested that perceptual closure is not an all-ornone phenomenon but is better described as a continuum (Elder \& Zucker, 1993, 1994; Gillam, 1975).

A number of studies have also demonstrated the effect of the distribution of gaps along the contours on perceptual closure and its utility in shape perception. Spehar (2002) showed that reversals in contrast polarity along bounding contours have a detrimental effect on the efficiency of search for a concave target among convex distractors when the reversals occur at points of high degree of change in contour direction, but not when they occur at straight contour segments. Of particular relevance to the present work, Kimchi (2000) showed that when gaps occur at the corners of a "square" configuration, the configuration of spatially close line segments, but not of distant line segments, is primed at brief exposures. When the gaps occur at the center of the "square" lines - namely, separating collinear

R. Kimchi, rkimchi@research.haifa.ac.il 
lines - the global configuration of both spatially close and spatially distant line segments is primed at brief exposures. The advantage of the combination of closure and collinearity over closure alone has been demonstrated in several other studies (e.g., Biederman, 1987; Donnelly, Humphreys, \& Riddoch, 1991; Pettet, McKee, \& Grzywacz, 1998). For example, Biederman showed that object identification is substantially hindered when the contours of objects are deleted at concavities, much more so than when the contours are deleted within straight segments, and Donnelly and colleagues (Donnelly et al., 1991) showed that target search is more efficient when distractors are grouped by closure and collinearity than by closure alone.

These findings suggest that studying the role of closure in grouping of shape should consider both the size of the gaps between the closure-inducing contours and their distribution along the contours.

The present study examined the time course of grouping line segments into a shape by closure as a function of spatial proximity and collinearity between the closureinducing line segments, using the primed matching paradigm (Beller, 1971). In this paradigm, observers are presented with a priming stimulus followed immediately by two test stimuli to match for identity. Reaction times (RTs) to correctly identified same test pairs depend on the representational similarity between the test stimuli and the prime: Responses are faster when the test stimuli are similar to the prime than when they are dissimilar to it (Beller, 1971; Rosch, 1975). Varying the exposure duration of the prime enables us to tap earlier and later internal representations (e.g., Kimchi, 1998, 2000; Sekuler \& Palmer, 1992).

The priming stimuli in the present study were line segments grouped by closure into spindle-like shapes. The spatial proximity between the closure-inducing line seg- ments varied (i.e., small vs. large gaps), and depending on the distribution of the gaps along the closure-inducing lines, collinearity was either present (collinear prime) or absent (noncollinear prime; see Figure 1). The primes were presented for various durations. There were two types of sameresponse test pairs defined by the similarity relation between the prime and the test stimuli. In the shape-similarity test pairs, the two stimuli were similar to the prime in shape, but dissimilar in line components. In the component-similarity test pairs, the stimuli were similar to the prime in line components but dissimilar in shape. For this set of stimuli, priming effects of the shape would manifest in faster responses to the shape-similarity test pairs than to the componentsimilarity test pairs, resulting from facilitation to the former (due to similarity in shape) and/or interference to the latter (due to dissimilarity in shape). Priming effects of the line components would manifest in faster responses to the component-similarity test pairs than to the shape-similarity ones, resulting from facilitation to the former (due to similarity in line components) and/or interference to the latter (due to dissimilarity in line components).

If grouping of shape by closure depends only on the gap size between the image fragments (e.g., Elder \& Zucker, 1993), then spatial proximity (small vs. large gap) between the closure-inducing contours should have a similar effect on the time course of grouping regardless of the distribution of the gaps along the closure-inducing contours - namely, regardless of whether collinearity is present or absent in the stimulus. If, however, grouping of shape by closure is also sensitive to the distribution of the gaps along the image fragments (i.e., whether the gaps occur at straight contour segments or at points of a high degree of change in contour direction; e.g., Kimchi, 2000; Spehar, 2002), then the effect of spatial proximity on the time course of grouping may depend on whether or not collinearity is present or absent.
A

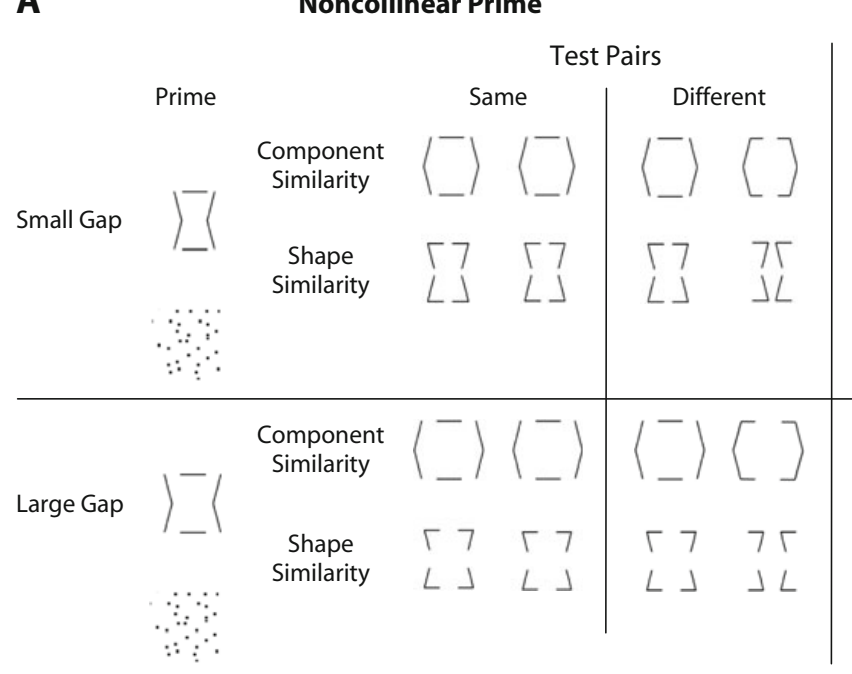

B

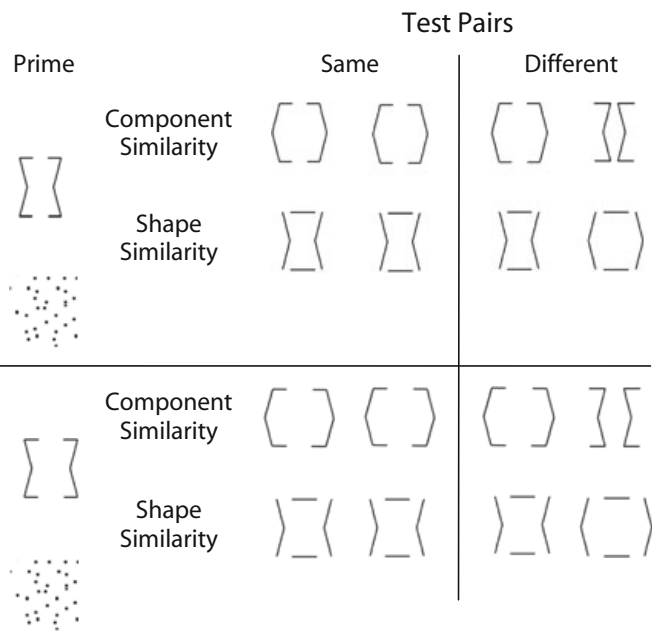

Figure 1. The primes and the same- and different-response test pairs for the (A) noncollinear and (B) collinear primes used in Experiment 1 . The complete set of primes and test pairs was also presented in a $90^{\circ}$ rotation. Each of the primes (a spindle-like shape or a random array of dots - the neutral prime) was followed by either the shape-similarity test pair or the component-similarity test pair. 
In particular, if the combination of closure and collinearity dominates spatial proximity in early perceptual organization, as suggested by Kimchi (2000), then early priming of the shape would be expected both when the closureinducing lines are spatially close (small gap) and spatially distant (large gap) for the collinear stimuli, but only when they are spatially close for the noncollinear stimuli.

\section{EXPERIMENT 1}

\section{Method}

Participants. Seventy-two students at the University of Haifa, 18 in each condition (noncollinear-small gap, noncollinear-large gap, collinear-small gap, and collinear-large gap), participated in this experiment. All participants had normal or corrected-to-normal vision. Participants received course credit or were paid for their participation.

Apparatus and Stimuli. Stimuli presentation and data collection were controlled by a Silicon Graphics O2 workstation. The screen was viewed through the circular aperture $(16 \mathrm{~cm}$ in diameter) of a matte black cardboard sheet. Responses were made by pressing one of two response keys, and RTs were recorded by the computer. The testing room was dimly lit.

Figure 1 depicts the primes and the corresponding same- and different-response test pairs for each condition. The priming stimuli were disconnected spindle-like shapes of two types, generated from a basic spindle-like shape composed of two bent lines and two connecting lines. The noncollinear prime was generated by removing the end portions of the connecting lines (Figure 1A). The collinear prime was generated by removing the central portion in each of the two connecting lines so that the remaining portions of each connecting line were collinear (Figure 1B). The gaps between the line segments were either small (spatially close line segments) or large (spatially distant line segments). A random array of dots was used as a neutral prime and served as a control condition.

There were two types of same-response test pairs. The componentsimilarity test pair consisted of two stimuli that had the same line segments as the prime, spatially rearranged to form a barrel-like shape. The shape-similarity test pair consisted of two stimuli that were similar to the prime in shape (i.e., had a spindle-like shape), but differed in line components (Figure 1). One different-response test pair included two stimuli that differed from one another in shape (a spindle-like shape vs. a barrel-like shape), and the other test pair included two barrel-like shapes that differed from one another in line components. The complete set of primes and test pairs was also presented in a $90^{\circ}$ rotation. Observers sat $70 \mathrm{~cm}$ from the screen with their heads resting on a chinrest. From this position, the stimuli subtended $1.47^{\circ} \times 1.88^{\circ}$ and $1.97^{\circ} \times 1.88^{\circ}$ for the small-gap and the large-gap conditions, respectively. For each gap condition, the total gap size was the same for the noncollinear and the collinear primes. Also, for each prime type, the size of the line segments was kept the same in the two gap conditions. The gaps between the lines in the noncollinear stimuli subtended $0.24^{\circ}$ each in the small-gap condition, and $0.49^{\circ}$ each in the large-gap condition. The gaps in the collinear stimuli subtended $0.48^{\circ}$ each in the small-gap condition, and $0.98^{\circ}$ each in the large-gap condition. The priming stimulus was presented at fixation; the test stimuli were presented to the sides of fixation, and the distance between the centers of the two stimuli was $7 \mathrm{~cm}$

Design. The experiment consisted of the factorial combination of seven factors in a mixed design: gap (small, large); prime type (noncollinear, collinear); priming condition (prime, control); prime-test similarity (shape similarity, component similarity); response (same, different); prime duration (30, 80, 180, 380, or $680 \mathrm{msec})$; and orientation $\left(0^{\circ}, 90^{\circ}\right)$. Gap and prime type were between-subjects factors, and the other five factors were within-subjects factors. Orientation was administered between blocks, and the other four factors were

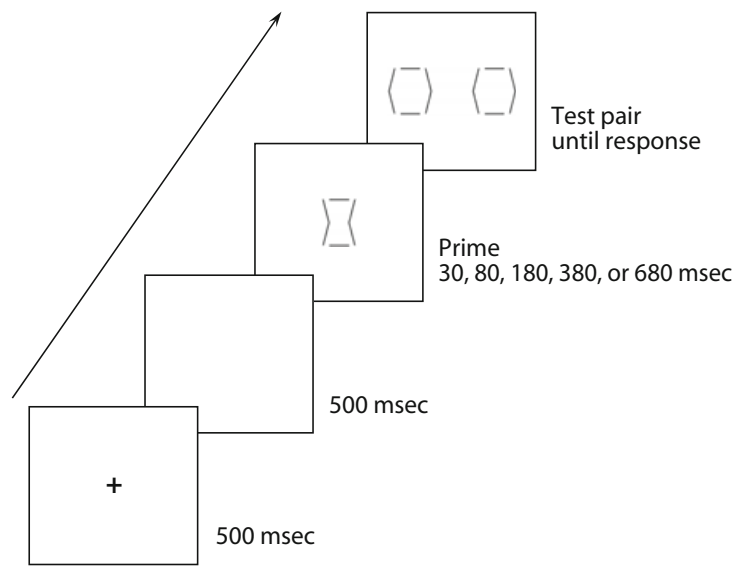

Figure 2. The sequence of events in a trial. The example depicts a noncollinear prime followed by a component-similarity test pair.

administered within blocks, with each combination occurring on an equal number of trials. For each of the four combinations of gap and prime type there were 960 experimental trials in six blocks of 160 trials each, preceded by a practice block of 24 trials.

Procedure. The sequence of events in each trial is depicted in Figure 2. First, a fixation point appeared in the center of the screen for $500 \mathrm{msec}$, followed by a 500 -msec interval. Then, the priming stimulus (a spindle-like shape in the prime condition, or an array of random dots in the control condition) appeared for a variable duration, followed immediately by the test pair. The test pair remained on the screen until the observer's response, for a maximum of 3,000 msec. Observers were asked to indicate, as quickly and accurately as possible, whether the two stimuli in the test pair were the same as each other or different from one another by pressing one of two response keys. All observers pressed the same response key with their dominant hand. Feedback about an incorrect response was provided by presenting an auditory tone as soon as the participant responded. Error trials were retaken up to three times at the end of the block.

\section{Results and Discussion}

All RT summaries and analyses are based on participants' mean RTs for correct same responses. We restricted the analysis to same responses because typically there is no effect of priming on different responses (e.g., Beller, 1971; Kimchi, 1998; Sekuler \& Palmer, 1992). RTs that fell $2.8 S D$ s above or below the mean of each participant ( $2.18 \%$ of all observations) were trimmed. Error rates were low (an overall mean of $2 \%$ ), and there was no indication of speed-accuracy trade-offs. Therefore, error rates are not discussed further. Mean RTs for the shape- and component-similarity test pairs for each prime type, as a function of gap, prime duration, and priming condition, are presented in Table 1.

The main dependent variable was priming. The priming measure, calculated for each prime type, indicates how much the prime in question speeded same responses to shape-similarity test pairs relative to component-similarity test pairs. The amount of priming is defined by the difference in same-response RT to a component-similarity test pair in comparison with a shape-similarity test pair after participants view the prime minus the baseline response 
Table 1

Mean RTs (in Milliseconds) for Shape- and Component-Similarity Test Pairs for Each Prime Type (Noncollinear, Collinear) As a Function of Gap (Small, Large), Priming Condition (Prime, Control), and Prime Duration in Experiment 1

\begin{tabular}{|c|c|c|c|c|c|c|c|c|}
\hline \multirow{3}{*}{$\begin{array}{c}\text { Prime } \\
\text { Duration } \\
(\mathrm{msec})\end{array}$} & \multicolumn{4}{|c|}{ Small Gap } & \multicolumn{4}{|c|}{ Large Gap } \\
\hline & \multicolumn{2}{|c|}{$\begin{array}{c}\text { Shape } \\
\text { Similarity }\end{array}$} & \multicolumn{2}{|c|}{$\begin{array}{l}\text { Component } \\
\text { Similarity }\end{array}$} & \multicolumn{2}{|c|}{$\begin{array}{c}\text { Shape } \\
\text { Similarity }\end{array}$} & \multicolumn{2}{|c|}{$\begin{array}{l}\text { Component } \\
\text { Similarity }\end{array}$} \\
\hline & Prime & Control & Prime & Control & Prime & Control & Prime & Control \\
\hline \multicolumn{9}{|c|}{ Noncollinear } \\
\hline 30 & 729 & 747 & 782 & 743 & 693 & 675 & 672 & 660 \\
\hline 80 & 732 & 746 & 772 & 768 & 685 & 681 & 656 & 675 \\
\hline 180 & 744 & 746 & 746 & 755 & 687 & 675 & 648 & 665 \\
\hline 380 & 761 & 754 & 753 & 760 & 690 & 690 & 658 & 689 \\
\hline 680 & 740 & 741 & 776 & 767 & 694 & 692 & 666 & 691 \\
\hline \multicolumn{9}{|c|}{ Collinear } \\
\hline 30 & 647 & 633 & 630 & 611 & 680 & 693 & 621 & 612 \\
\hline 80 & 633 & 636 & 631 & 604 & 662 & 684 & 641 & 613 \\
\hline 180 & 612 & 633 & 633 & 587 & 690 & 681 & 623 & 613 \\
\hline 380 & 622 & 618 & 629 & 583 & 679 & 671 & 618 & 599 \\
\hline 680 & 601 & 623 & 610 & 585 & 689 & 683 & 625 & 612 \\
\hline
\end{tabular}

difference for these test pairs in the control condition (Equation 1; see also Guttman, Sekuler, \& Kellman, 2003).

$$
\begin{aligned}
\text { Priming }= & {[\mathrm{RT}(\text { component similarity } / \text { prime })} \\
& -\mathrm{RT}(\text { shape similarity } / \text { prime })] \\
- & {[\mathrm{RT}(\text { component similarity/control })} \\
& -\mathrm{RT}(\text { shape similarity } / \text { control })] .
\end{aligned}
$$

Priming of the shape should produce priming values of greater than zero, caused by an advantage in responding to the shape-similarity test pairs over the component-similarity test pair in the prime condition relative to any RT difference between these test pairs in the control condition. Priming of the line components, on the other hand, should produce priming values of less than zero, caused by an advantage in responding to the component-similarity test pair over the shape-similarity test pair in the prime condition relative to the control condition. Note that because each test pair is similar to the prime in one aspect (e.g., shape) and dissimilar in the other aspect (e.g., components), the priming effect can result from facilitation, inhibition, or a combination of both. Therefore, the observed priming effects actually indicate relative dominance of either shape or components.

Figure 3 depicts the priming effects for each prime type as a function of gap and prime duration. Preliminary analysis showed no significant effect of orientation and no interactions involving this factor. Therefore, the collapsed data were submitted to a three-factor (gap $\times$ prime type $\times$ prime duration) ANOVA, with gap and prime type as between-subjects factors and prime duration as a withinsubjects factor. The analysis revealed significant effects of prime type $[F(1,68)=18.00, p<.0001]$ and gap $[F(1,68)=12.44, p<.0008]$, and a significant interaction between prime type and prime duration $[F(4,272)=$ $3.16, p<.02]$. Most importantly, the analysis yielded a significant interaction between gap, prime type, and prime duration $[F(4,272)=3.01, p<.02]$.

Planned comparisons were conducted to determine the effect of gap size under the short range of prime dura- tion (30 and $80 \mathrm{msec})$ and the longer range $(180,380$, and $680 \mathrm{msec}$ ) for each prime type. Reliability of priming values (greater than zero or less than zero) relied on $t$ tests with $\alpha=.05$. The analysis for the noncollinear prime (Figure $3 \mathrm{~A}$ ) revealed a significant difference between small and large gap under the shorter range of prime duration $[F(1,34)=$ $13.86, p<.0007]$. A priming value greater than zero $(M=$ $37 \mathrm{msec}$ ), indicating priming of the shape, was observed for
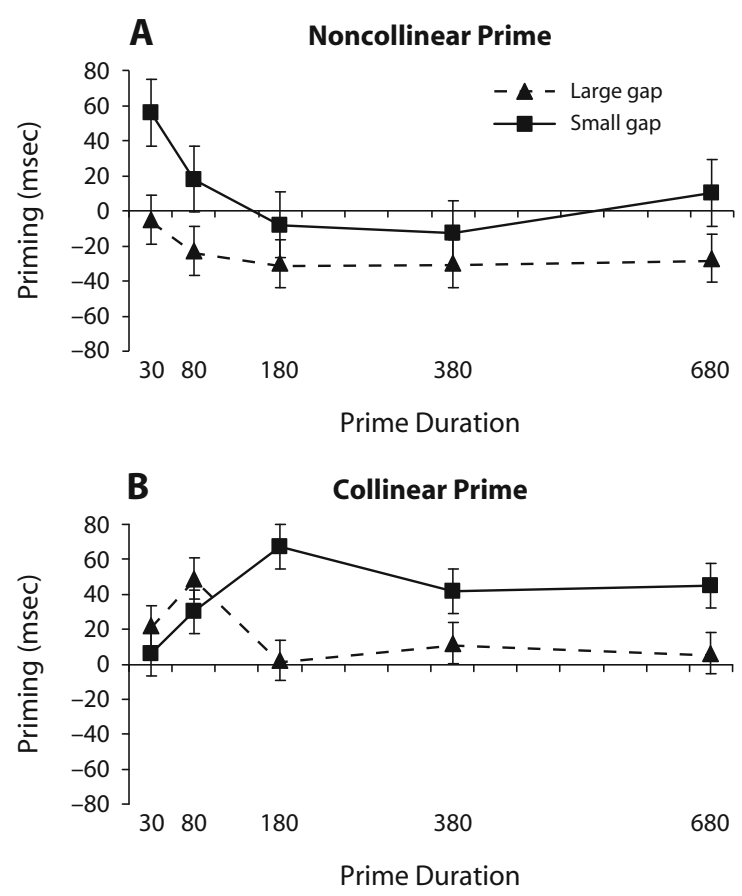

Figure 3. Results for Experiment 1: Priming effects for (A) noncollinear and (B) collinear primes as a function of gap and prime duration. Values greater than zero indicate shape priming; values less than zero indicate components priming (see text for details). 
A

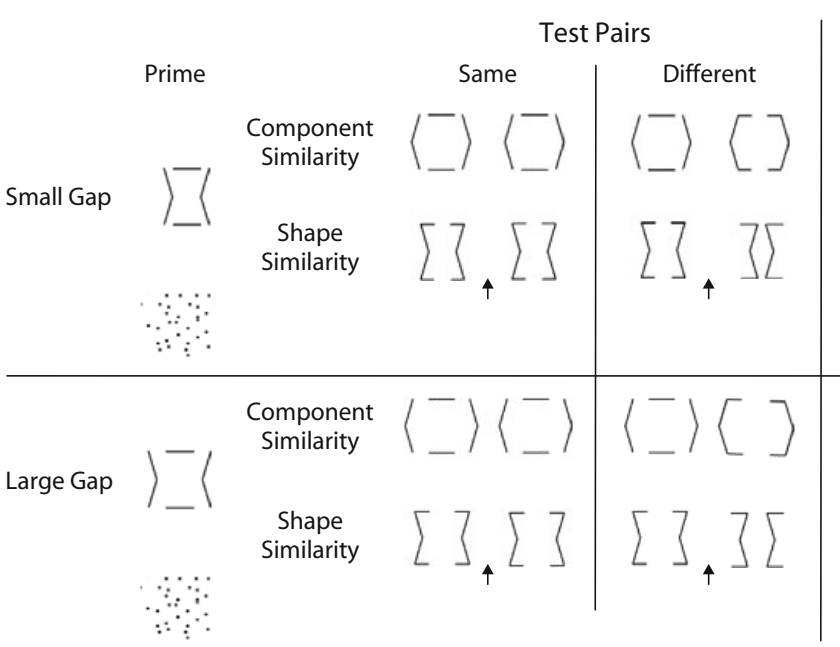

B

Collinear Prime

Figure 4. The primes and the same- and different-response test pairs for (A) noncollinear and (B) collinear primes, used in Experiment 2. This set of stimuli is the same as in Experiment 1, except that no gaps occur at the centers of the vertical contours in the shapesimilarity test stimuli (the small arrows point to the test pairs that differ from the ones used in Experiment 1).

the small-gap condition, whereas a tendency for priming value less than zero $(M=-14 \mathrm{msec}$, n.s.) was observed for the large gap. For the longer range of prime duration, the effect of gap approached significance $[F(1,34)=2.87, p<$ $.09]$. A priming value less than zero $(M=-30 \mathrm{msec})$, indicating priming of the component lines, was observed for the large-gap condition; no significant priming $(M=-4 \mathrm{msec}$, n.s.) was observed for the small-gap condition.

A different pattern of results was observed for the collinear prime (Figure 3B). The analysis for the shorter range of prime duration showed no significant effect of gap $[F(1,34)=1.77, p>.19]$. A priming value greater than zero, indicating shape priming, was observed for both gap conditions, though the priming value reached statistical significance for the large-gap condition $(M=$ $36 \mathrm{msec})$, but not for the small-gap condition $(M=$ $18 \mathrm{msec}$, n.s.). The analysis for the longer range of prime duration showed a significant effect of gap $[F(1,34)=$ $6.83, p<.02]$. A reliable shape priming remained evident for the small gap $(M=52 \mathrm{msec})$, but no significant priming was observed for the large gap ( $M=7 \mathrm{msec}$, n.s.), indicating that shape priming attenuated with time when the line components were spatially distant.

These results show that in the absence of collinearity, the early grouping of shape by closure is facilitated by spatial proximity: Shape was primed under short exposure durations when the closure-inducing line segments were spatially close, but not when they were spatially distant. In the presence of collinearity, priming of the shape was observed under short exposure durations both for spatially close and spatially distant line segments, indicating the early grouping of the line segments into a shape, regardless of the spatial proximity between the closure-inducing segments. However, the relative dominance of shape persisted over time only when the closure-inducing lines were spatially close.
In the present experiment, we attempted as much as possible to keep the same number of line segments in the prime and in the test stimuli to avoid introducing irrelevant dissimilarity between the prime and the test stimuli. In doing so, we positioned the gaps in the shape-similarity test stimuli in the noncollinear condition at the center of the bent lines (i.e., at points of highest contour curvature; see Figure 1A), possibly hindering the appearance of the shape of these test stimuli. This was not the case, however, for the shapesimilarity test stimuli in the collinear condition (Figure 1B). It is possible, then, that the degree of representational similarity between the noncollinear prime and its corresponding shape-similarity test stimuli was lower than that between the collinear prime and its corresponding shape-similarity test stimuli. Therefore, one may argue that the differential effect of spatial proximity on priming for the two prime types is accounted for by differences in the degree of representational similarity between the primes and their corresponding shape-similarity test stimuli rather than by the difference between the two primes in the presence versus absence of collinearity. The following experiment was conducted to rule out this alternative account. Experiment 2 basically used the same set of stimuli, only now the gaps did not occur in any case at the centers of the vertical contours in the shapesimilarity test stimuli (see Figure 4).

\section{EXPERIMENT 2}

\section{Method}

Participants. Seventy-nine new students at the University of Haifa participated in this experiment: 20 in the noncollinear-smallgap condition, 15 in the noncollinear-large-gap condition, 21 in the collinear-small-gap condition, and 23 in the collinear-large-gap condition.

Stimuli. Figure 4 depicts the primes and the corresponding sameand different-response test pairs for each condition. The stimuli mea- 
Table 2

Mean RTs (in Milliseconds) for Shape- and Component-Similarity Test Pairs for Each Prime Type (Noncollinear, Collinear) As a Function of Gap (Small, Large), Priming Condition (Prime, Control), and Prime Duration in Experiment 2

\begin{tabular}{|c|c|c|c|c|c|c|c|c|}
\hline \multirow{3}{*}{$\begin{array}{c}\text { Prime } \\
\text { Duration } \\
(\mathrm{msec})\end{array}$} & \multicolumn{4}{|c|}{ Small Gap } & \multicolumn{4}{|c|}{ Large Gap } \\
\hline & \multicolumn{2}{|c|}{$\begin{array}{c}\text { Shape } \\
\text { Similarity }\end{array}$} & \multicolumn{2}{|c|}{$\begin{array}{l}\text { Component } \\
\text { Similarity }\end{array}$} & \multicolumn{2}{|c|}{$\begin{array}{c}\text { Shape } \\
\text { Similarity }\end{array}$} & \multicolumn{2}{|c|}{$\begin{array}{l}\text { Component } \\
\text { Similarity }\end{array}$} \\
\hline & Prime & Control & Prime & Control & Prime & Control & Prime & Control \\
\hline \multicolumn{9}{|c|}{ Noncollinear } \\
\hline 30 & 748 & 733 & 783 & 737 & 774 & 748 & 741 & 754 \\
\hline 80 & 718 & 745 & 743 & 752 & 759 & 730 & 722 & 756 \\
\hline 180 & 744 & 765 & 734 & 746 & 770 & 764 & 749 & 736 \\
\hline 380 & 761 & 767 & 747 & 765 & 765 & 754 & 713 & 735 \\
\hline 680 & 735 & 732 & 751 & 773 & 752 & 739 & 715 & 738 \\
\hline \multicolumn{9}{|c|}{ Collinear } \\
\hline 30 & 694 & 703 & 676 & 659 & 751 & 774 & 684 & 686 \\
\hline 80 & 685 & 706 & 673 & 650 & 730 & 751 & 681 & 676 \\
\hline 180 & 694 & 683 & 678 & 651 & 756 & 758 & 680 & 679 \\
\hline 380 & 681 & 686 & 669 & 632 & 752 & 751 & 668 & 669 \\
\hline 680 & 682 & 684 & 678 & 644 & 744 & 746 & 661 & 685 \\
\hline
\end{tabular}

surements were the same as in Experiment 1. The only difference was that no gaps were positioned in the centers of the vertical bent lines.

Design and Procedure. All aspects of the design and procedure were the same as in Experiment 1.

\section{Results and Discussion}

RT summaries and analyses are based on participants' mean RTs for correct same responses. RTs that fell 2.8 SDs above or below the mean of each participant $(2.16 \%$ of all observations) were trimmed. Error rates were low (an overall mean of $1.8 \%$ ), and there was no indication of speedaccuracy trade-offs. Mean RTs for shape- and componentsimilarity test pairs for each prime type as a function of prime duration and gap are presented in Table 2 .

Figure 5 depicts the priming effects (calculated as in Experiment 1) for each prime type as a function of gap and prime duration. A three-factor $($ gap $\times$ prime type $\times$ prime duration) ANOVA was performed on the priming scores, with gap and prime type as between-subjects factors and prime duration as a within-subjects factor. The analysis revealed significant effects of prime type $[F(1,75)=15.72$, $p<.0002]$ and gap $[F(1,75)=14.93, p<.0002]$ and a significant interaction between prime type, gap, and prime duration $[F(4,300)=2.54, p<.04]$.

As in Experiment 1, planned comparisons were conducted to determine the effect of gap size under the short range (30 and $80 \mathrm{msec})$ and the longer range $(180,380$, and $680 \mathrm{msec}$ ) of prime duration, for each prime type. The analysis for the noncollinear prime (Figure 5A) revealed a significant effect of gap under the shorter range of prime duration $[F(1,33)=16.81, p<.0003]$. The noncollinear prime produced priming values greater than zero $(M=$ $25 \mathrm{msec}$ ), indicating priming of the shape, in the smallgap condition, and priming values less than zero $(M=$ $-50 \mathrm{msec}$ ), indicating priming of the line components, in the large-gap condition. No significant effect of gap was obtained for the longer range of prime duration $(F<1)$. Priming values less than zero were observed for both gap conditions, although reliable components priming was evident for the large gap $(M=-20 \mathrm{msec})$ but not for the small gap $(M=-9 \mathrm{msec}$, n.s. $)$.

A different pattern of results was observed for the collinear prime (Figure 5B). No effect of gap was observed under brief exposures of the prime $(F<1)$. Collinear primes produced priming values greater than zero both in the small-gap condition $(M=35 \mathrm{msec})$ and in the largegap condition $(M=24 \mathrm{msec})$, indicating the early orga-
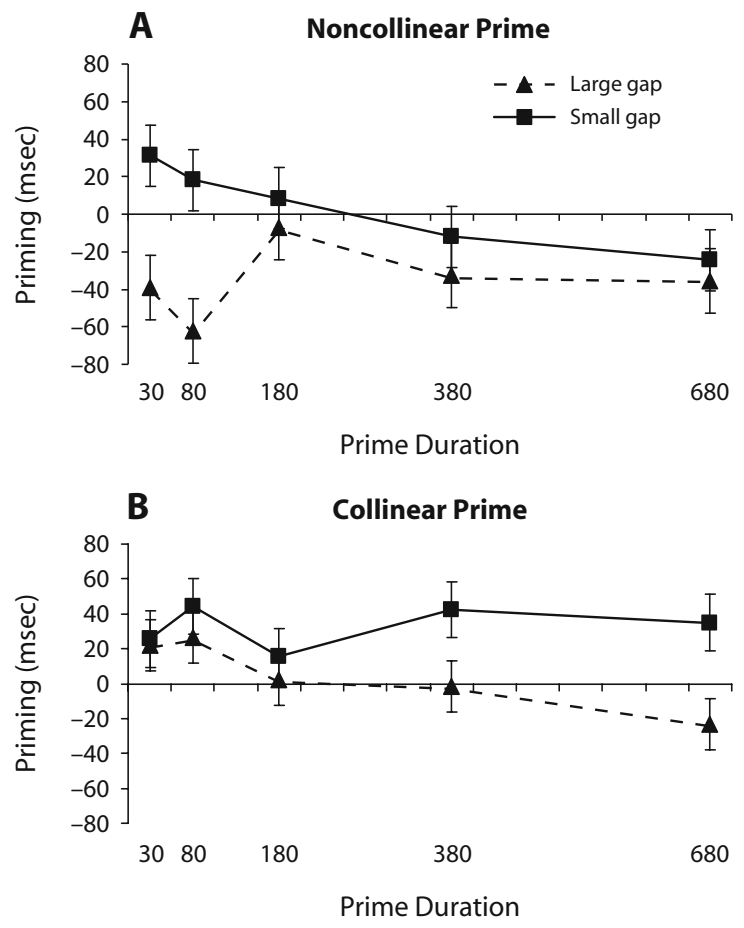

Figure 5. Results for Experiment 2: Priming effects for the (A) noncollinear and (B) collinear primes, as a function of gap and prime duration. 
A

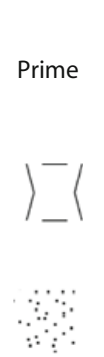

Noncollinear Prime

Test Pairs
B

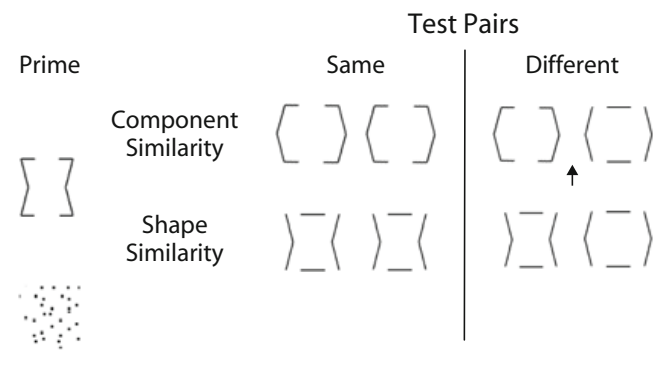

Figure 6. The primes and the same- and different-response test pairs for the (A) noncollinear and (B) collinear primes used in Experiment 3. Stimuli used in this experiment are identical to those used in Experiment 2 , except for the different-response test pairs (the small arrows point to the test pairs that were changed). This control experiment used only spatially distant line segments.

nization of the line segments into a shape, regardless of gap size. Gap had a significant effect on priming under the longer range of prime duration $[F(1,42)=6.37, p<$ .02]. A reliable shape priming remained evident for the small gap $(M=31 \mathrm{msec})$, but not for the large gap $(M=$ $-8 \mathrm{msec}$, n.s.). Thus, the relative dominance of the global shape appeared to attenuate over time when the line components were spatially distant.

The pattern of results in this experiment was similar to the one observed in Experiment 1. The present results for the noncollinear condition demonstrated again the critical role of spatial proximity: Shape was primed under short exposure durations only when the closure-inducing line segments were spatially close, but not when they were spatially distant. The results for the collinear condition were also replicated: Priming of the shape was observed both for spatially close and spatially distant line segments under the short exposure durations. These results suggest that it is the absence/presence of collinearity that was responsible for the difference in priming effects produced by the noncollinear versus the collinear primes in the large-gap condition in Experiment 1, rather than the potential difference in the degree of representational similarity between the primes and their corresponding shape-similarity test stimuli.

A consistent result observed in Experiments 1 and 2 is that although collinear line segments produced shape priming under short prime exposures regardless of spatial proximity between the line segments, only spatially close, but not spatially distant, collinear line segments continued to produce reliable shape priming under longer prime exposures (180 $\mathrm{msec}$ and longer). The finding that the organization of collinear line segments is available for priming under brief exposures, regardless of their spatial proximity, suggests that grouping by collinearity is fast occurring and overrides poor proximity. Previous studies provide psychophysical and electrophysiological support to this claim, also arguing that perceptual grouping by collinearity is accomplished by early visual processes (e.g., Behrmann \& Kimchi, 2003; Kimchi, 2000; Lee, 2003; Sugita, 1999). Our results, however, indicate that the relative dominance of the shape of collinear line segments attenuates with time when the lines are spatially distant. This suggests that when presented long enough, spatial proximity between the lines does play a role, perhaps because it influences the cohesion of the shape, resulting in accessibility of the representation of the closure-inducing line segments.

A final reservation to consider concerns the differentresponse test pairs. As described above, one differentresponse test pair contains two test stimuli that are different from one another in shape but have the same components, whereas the other pair contains two test stimuli that have similar components but different shapes. However, in the collinear condition, the different-response test pair in which the two stimuli have different components actually includes two stimuli that also appear to have different shapes (see Figure 4B). Consequently, the two differentresponse test pairs that followed the collinear prime contained a pair of stimuli that differ in shapes. This might have biased responses toward the shape in the collinear condition. Therefore, a control experiment was conducted to confirm that it is the presence of collinearity that underlies the rapid organization of the collinear prime into a shape in the large-gap condition, rather than a potential bias toward the shape. Experiment 3 reexamined priming effects for spatially distant line segments as a function of prime type (noncollinear vs. collinear), using the set of stimuli depicted in Figure 6.

\section{EXPERIMENT 3}

\section{Method}

Participants. Thirty-five new participants were tested in this control experiment, 19 in the noncollinear condition and 16 in the collinear condition. All participants had normal or corrected-tonormal vision. Participants received course credit or were paid for their participation.

Stimuli, Design, and Procedure. Figure 6 depicts the primes and the corresponding same- and different-response test pairs for each prime type. The only difference between the stimuli used in this experiment and those used in Experiment 2 concerned the differentresponse test pairs. In the present experiment, we ensured that when the two stimuli in the different-response test pair have different components they have similar shape. All other aspects of stimuli, design, 
and procedure were the same as in Experiment 2, except that only the large-gap condition was employed in this experiment.

\section{Results and Discussion}

RT summaries and analyses are based on participants' mean RTs for correct same responses. Again, RTs that fell $2.8 \mathrm{SDs}$ above or below the mean of each participant (2.19\% of all observations) were trimmed. Error rates were low (an overall mean of $1.9 \%$ ), and there was no indication of speed-accuracy trade-offs. Therefore, error rates are not discussed further. Mean RTs to shape- and component-similarity test pairs for each prime type as a function of prime duration and gap are presented in Table 3. RTs in the present experiment were somewhat higher than in Experiments 1 and 2, presumably because the different-response test pairs were more difficult to discriminate. Other than that, the pattern of results for spatially distant line segments was replicated.

Figure 7 depicts the priming effects (calculated as in Experiments 1 and 2) for each prime type as a function of prime duration. Planned comparisons were conducted to examine the difference between the prime types for the short range (30 and $80 \mathrm{msec}$ ) and the longer range (180, 380 , and $680 \mathrm{msec}$ ) of prime duration. The analysis for the short range of duration revealed a significant effect of prime type $[F(1,33)=7.57, p<.01]$. The collinear prime produced priming values greater than zero $(M=$ $38 \mathrm{msec}$ ), indicating shape priming, whereas no significant priming ( $M=-4 \mathrm{msec}$, n.s.) was produced by the noncollinear prime. Under the longer range of prime duration, the noncollinear prime produced priming values less than zero $(M=-18 \mathrm{msec})$, indicating priming of the components; no significant priming was observed for the collinear prime $(M=-11 \mathrm{msec}$, n.s.). These results are congruent with the results of the two previous experiments for the large-gap condition. Under the short range of prime duration, only the collinear prime produced reliable shape priming. Under the longer range of prime duration, the collinear prime produced unreliable priming values whereas the noncollinear prime produced reliable components priming.

These results suggest that it is the presence of collinearity that is responsible for the difference in priming effects produced by the noncollinear versus the collinear primes in the large-gap condition in Experiments 1 and 2, rather
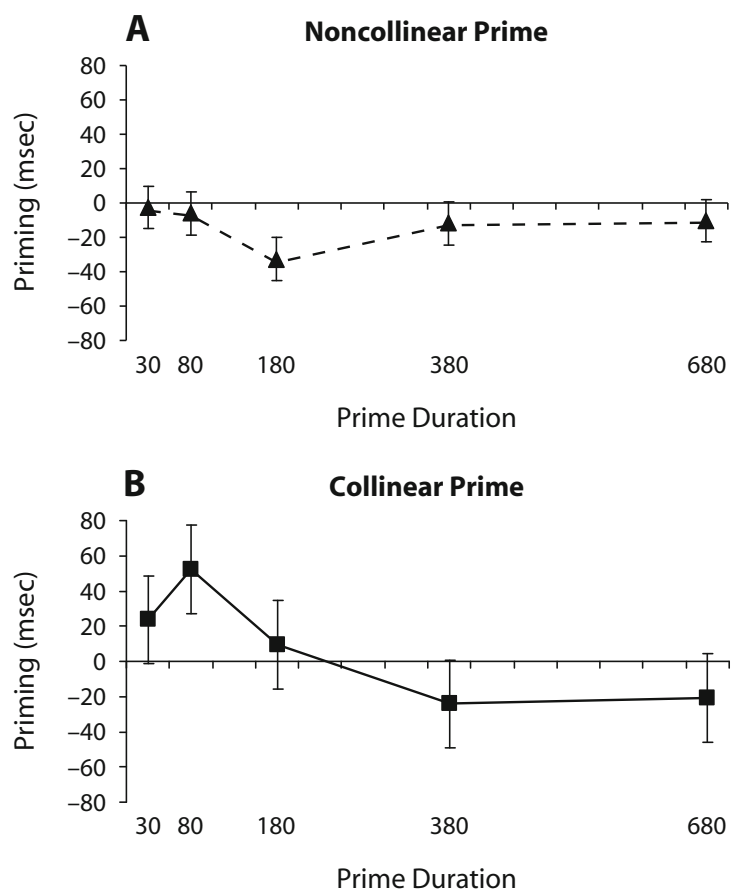

Figure 7. Results for Experiment 3: Priming effects for the (A) noncollinear and (B) collinear primes, as a function of prime duration.

than any potential bias toward the shape that the differentresponse test pairs of the collinear condition might have caused.

\section{GENERAL DISCUSSION}

The present results show that spatial proximity between the closure-inducing line segments was critical for the rapid grouping into a shape in the absence of collinearity, but not in the presence of collinearity. These results converge with Kimchi's (2000) microgenetic results in demonstrating that the combination of closure and collinearity dominates proximity in early perceptual organization. The present study, however, extends Kimchi's (2000) study by demonstrating the role of closure and its interac-

Table 3

Mean RTs (in Milliseconds) for Configuration- and Component-Similarity Test Pairs for Each Prime Type As a Function of Priming Condition (Prime, Control) and Prime Duration in Experiment 3

\begin{tabular}{|c|c|c|c|c|c|c|c|c|}
\hline \multirow{3}{*}{$\begin{array}{l}\text { Prime } \\
\text { Duration } \\
(\mathrm{msec})\end{array}$} & \multicolumn{4}{|c|}{ Noncollinear Prime } & \multicolumn{4}{|c|}{ Collinear Prime } \\
\hline & \multicolumn{2}{|c|}{$\begin{array}{c}\text { Shape } \\
\text { Similarity }\end{array}$} & \multicolumn{2}{|c|}{$\begin{array}{l}\text { Component } \\
\text { Similarity }\end{array}$} & \multicolumn{2}{|c|}{$\begin{array}{c}\text { Shape } \\
\text { Similarity }\end{array}$} & \multicolumn{2}{|c|}{$\begin{array}{l}\text { Component } \\
\text { Similarity }\end{array}$} \\
\hline & Prime & Control & Prime & Control & Prime & Control & Prime & Contro \\
\hline 30 & 746 & 724 & 765 & 743 & 815 & 797 & 790 & 749 \\
\hline 80 & 719 & 715 & 751 & 745 & 804 & 817 & 773 & 731 \\
\hline 180 & 743 & 723 & 733 & 747 & 804 & 806 & 767 & 760 \\
\hline 380 & 758 & 739 & 760 & 753 & 816 & 815 & 747 & 771 \\
\hline 680 & 755 & 729 & 762 & 740 & 801 & 789 & 741 & 750 \\
\hline
\end{tabular}

Note-Only large gaps were employed in this experiment. 
tion with proximity and collinearity in the early grouping of shape. Using the primed matching procedure, Kimchi (2000) presented observers with a prime that possessed closure (e.g., a square made of lines) followed either by a shape-similarity test pair (e.g., squares made of different line components than the prime), or by a componentsimilarity test pair (e.g., crosses made of the same lines as the prime), while varying the gaps between the closureinducing segments of the prime and the prime duration. Thus, the shape-similarity test stimuli were closed shapes, whereas the component-similarity test stimuli were open ones. Therefore, the advantage in responding to the shapesimilarity test pairs in Kimchi's (2000) experiment demonstrated the availability of closure and its interaction with proximity and collinearity in early perceptual organization, but not necessarily the grouping of shape by closure. In the present study, however, both the shape-similarity and component-similarity test stimuli are "closed" shapes that differ in their specific shape, and therefore, the advantage in responding to the shape-similarity test pairs can be reasonably attributed to shape representation. Thus, the present results demonstrate the role of closure and its interaction with proximity and collinearity in the early grouping of shape, rather than just the availability of closure in early perceptual organization.

The present results also converge nicely with previous results with adults in a visual search task (Hadad \& Kimchi, 2006). Observers in Hadad and Kimchi's study searched for a concave target among a variable number of convex distractors. The stimuli were similar to the ones used in the present experiment and varied in spatial proximity between the closure-inducing line segments and in the presence or absence of collinearity. When collinearity was absent in the stimuli, search for the shape of spatially close line segments was highly efficient, whereas the shape of spatially distant line segments was searched inefficiently. When collinearity was available, the shape of both spatially close and spatially distant line segments was searched efficiently. Thus, the shape of the noncollinear stimuli was primed at brief exposures and accessible to rapid search when the closure-inducing line segments were spatially close but not when they were spatially distant. The shape of the collinear stimuli, on the other hand, was primed at brief exposures and was efficiently searched both when the closure-inducing line segments were spatially close and spatially distant. Thus, the results of the present primed matching task and those of the visual search task converge in demonstrating that spatial proximity between closure-inducing lines influences rapid grouping into a shape for noncollinear stimuli, but has no effect on rapid grouping for collinear stimuli.

As noted earlier, the power of the combination of closure and collinearity over closure alone has been demonstrated previously in several studies (e.g., Donnelly et al., 1991; Kimchi, 2000; Spehar, 2002). The present results clearly demonstrate that the combination of closure and collinearity is particularly powerful for rapid grouping of shape when the closure-inducing line segments are not in close proximity.
The present results are generally consistent with the idea that perceptual closure is not an all-or-none phenomenon but rather a continuum (Elder \& Zucker, 1993, 1994; Gillam, 1975; Hadad \& Kimchi, 2006). However, the present results also show that the efficiency of grouping fragments into a shape by closure depends critically not only on the size of the gaps between the closure-inducing fragments, but also on the distribution of the gaps along the contours. Apparently, large gaps hinder the rapid organization of noncollinear closure-inducing lines (i.e., when gaps occur at point of change in contour direction) but not of collinear closure-inducing lines (i.e., when gaps occur at straight contour segments).

The finding that closure is highly sensitive to spatial proximity is not surprising, since the more spatially close the line segments are, the better the closure. The presence of collinearity, on the other hand, allows for rapid contour interpolation that appears to be less sensitive to spatial proximity within a certain range. These relations between closure, proximity, and collinearity in shape processing seem to be consistent with ecological considerations. The probability that noncollinear line segments compose the same object in a visual scene is not high, but it is much increased when these lines are spatially close. Spatial proximity should therefore determine the perceptual organization of these line segments. Collinear line segments, however, are better candidates for grouping into a coherent object because they are more likely to reflect portions of a real object's contour. Weak proximity should therefore be less detrimental for the early organization of these line segments. A rapid computation of collinearity between closure-inducing line segments that is insensitive to spatial proximity (within a certain range) enables efficient image descriptions and apparently is crucial for a fast, reliable interpretation of the visual scene in the real world. It should be noted, however, as mentioned earlier, that later in time the relative dominance of the shape over the line components decreased when the collinear closure-inducing lines were spatially distant, so that priming effects of shape persisted over time only when the closure-inducing fragments were collinear and spatially close. These findings suggest that a stable representation of shape over time depends both on spatial proximity and collinearity between the closure-inducing fragments.

Finally, a comparison between the present microgenetic results and the ontogenetic results of Hadad and Kimchi (2006) makes it possible to examine the relationship between microgenesis and ontogenesis of perceptual organization. A particular way of viewing the relation between these two time scales of visual perception was suggested by Werner $(1948,1957)$. According to Werner's proposal, there is an underlying unity in the pattern of change in perceptual development and in the emergence of a percept over time. Recent attempts to compare these two developmental courses of perceptual organization (Burack, Enns, Iarocci, \& Randolph, 2000; Enns, Burack, Iarocci, \& Randolph, 2000; Kimchi, Hadad, Behrmann, \& Palmer, 2005) appear to provide evidence for a link between ontogenetic and microgenetic change, demonstrating that the 
processes that exhibited different developmental trajectories were the ones identified by microgenetic analysis as differing in time course and attentional demands. For example, Kimchi (1998; Kimchi et al., 2005) showed that the rapid and effortless grouping of many small elements and the individuation of few large elements mature at a relatively early age, whereas the time-consuming and effortful grouping of few large elements and the individuation of many small elements develop with age. The present findings, however, taken together with those of Hadad and Kimchi (2006), suggest that there is no necessary correspondence between the two time scales of perceptual organization. The present microgenetic findings indicate rapid grouping of shape by closure combined with collinearity, both when the closure-inducing lines are spatially close and when they are spatially distant. The ontogenetic findings (Hadad \& Kimchi, 2006), on the other hand, indicate that only older children and adults (but not 5-year-olds) are able to use collinearity to enhance closure when the closure-inducing line segments are spatially distant, suggesting a longer developmental progression in the ability to employ long-range contour interpolation (see also Kovács, 2000; Kovács, Kozma, Fehér, \& Benedek, 1999). Thus, equally rapid groupings for adults can nevertheless show different developmental trajectories.

\section{AUTHOR NOTE}

This research was partly based on B.-S.H.'s dissertation under the supervision of R.K. This research was supported by the Max Wertheimer Minerva Center for Cognitive Processes and Human Performance, University of Haifa, and by a grant from the Research Authority, University of Haifa, to R.K. Facilities for conducting the research were provided by the Minerva Center and by the Institute of Information Processing and Decision Making, University of Haifa. We thank Hanna Strominger for programming assistance and Roni Raz for assistance in data collection. Correspondence concerning this article should be addressed to R. Kimchi, Department of Psychology, University of Haifa, Haifa 31905, Israel (e-mail: rkimchi@research.haifa.ac.il).

\section{REFERENCES}

Behrmann, M., \& Kimchi, R. (2003). What does visual agnosia tell us about perceptual organization and its relationship to object perception? Journal of Experimental Psychology: Human Perception \& Performance, 29, 19-42.

Beller, H. K. (1971). Priming: Effects of advance information on matching. Journal of Experimental Psychology, 87, 176-182.

Biederman, I. (1987). Recognition-by-components: A theory of human image understanding. Psychological Review, 94, 115-147.

Burack, J. A., Enns, J. T., Iarocci, G., \& Randolph, B. (2000). Age differences in visual search for compound patterns: Long- versus short-range grouping. Developmental Psychology, 36, 731-740.

Donnelly, N., Humphreys, G. W., \& Riddoch, M. J. (1991). Parallel computation of primitive shape descriptions. Journal of Experimental Psychology: Human Perception \& Performance, 17, 561-570.

ELDER, J. [H.], \& ZuCKER, S. [W.] (1993). The effect of contour closure on the rapid discrimination of two-dimensional shapes. Vision Research, 33, 981-991.
Elder, J. [H.], \& ZuCKER, S. [W.] (1994). A measure of closure. Vision Research, 34, 3361-3369.

ELDER, J. H., \& ZUCKER, S. W. (1998). Evidence for boundary-specific grouping. Vision Research, 38, 143-152.

Enns, J. T., Burack, J. A., Iarocci, G., \& Randolph, B. (2000). The orthogenetic principle in the perception of "forests" and "trees." Journal of Adult Development, 7, 41-48.

Gillam, B. (1975). New evidence for "closure" in perception. Perception \& Psychophysics, 17, 521-524.

Guttman, S. E., Sekuler, A. B., \& Kellman, P. J. (2003). Temporal variations in visual completion: A reflection of spatial limits? Journal of Experimental Psychology: Human Perception \& Performance, 29, 1211-1227.

HADAD, B.-S., \& KIMCHI, R. (2006). Developmental trends in utilizing perceptual closure for grouping of shape: Effects of spatial proximity and collinearity. Perception \& Psychophysics, 68, 1264-1273.

KIмcHI, R. (1998). Uniform connectedness and grouping in the perceptual organization of hierarchical patterns. Journal of Experimental Psychology: Human Perception \& Performance, 24, 1105-1118.

KIMCHI, R. (2000). The perceptual organization of visual objects: A microgenetic analysis. Vision Research, 40, 1333-1347.

Kimchi, R., Hadad, B.-S., Behrmann, M., \& Palmer, S. E. (2005). Microgenesis and ontogenesis of perceptual organization: Evidence from global and local processing of hierarchical patterns. Psychological Science, 16, 282-290.

KoffKa, K. (1935). Principles of Gestalt psychology. New York: Harcourt, Brace.

Kovács, I. (2000). Human development of perceptual organization. Vision Research, 40, 1301-1310.

KovÁcs, I., \& Julesz, B. (1993). A closed curve is much more than an incomplete one: Effect of closure in figure-ground segmentation. Proceedings of the National Academy of the Sciences, 90, 7495-7497.

Kovács, I., Kozma, P., Fehér, Á., \& BenedeK, G. (1999). Late maturation of visual spatial integration in humans. Proceedings of the $\mathrm{Na}$ tional Academy of Sciences, 96, 12204-12209.

LEE, T. S. (2003). Computations in the early visual cortex. Journal of Physiology, 97, 121-139.

Marino, A. C., \& SCHOLl, B. J. (2005). The role of closure in defining the "objects" of object-based attention. Perception \& Psychophysics, 67, 1140-1149.

Pettet, M. W., McKee, S. P., \& Grzywacz, N. M. (1998). Constraints on long range interactions mediating contour detection. Vision Research, 38, 865-879.

Rosch, E. (1975). Cognitive representations of semantic categories. Journal of Experimental Psychology: General, 104, 192-233.

SAarinen, J., \& LeVI, D. M. (1999). The effect of contour closure on shape perception. Spatial Vision, 12, 227-238.

Sekuler, A. B., \& Palmer, S. E. (1992). Perception of partly occluded objects: A microgenetic analysis. Journal of Experimental Psychology: General, 121, 95-111.

SpeHar, B. (2002). The role of contrast polarity in perceptual closure. Vision Research, 42, 343-350.

Sugita, Y. (1999). Grouping of image fragments in primary visual cortex. Nature, 401, 269-272.

WERnER, H. (1948). Comparative psychology of mental development. New York: International Universities Press.

WERnER, H. (1957). Comparative psychology of mental development (Rev. ed.). New York: International Universities Press.

Wertheimer, M. (1955). Gestalt theory. In W. D. Ellis (Ed.), A source book of Gestalt psychology (pp. 1-16). London: Routledge \& Kegan Paul. (Originally published in German, 1923)

(Manuscript received June 28, 2007; revision accepted for publication January 28, 2008.) 